\title{
iPoint: A Platform-independent Passive Information Kiosk for Cell Phones
}

\author{
Hooman Javaheri \\ College of Computer and Information Science \\ Northeastern University \\ Boston, MA 02115 \\ Email: hooman@ccs.neu.edu
}

\begin{abstract}
We introduce iPoint, a passive device that can interact and deliver information to virtually any mobile phone equipped with a WiFi network interface and a camera. The iPoint does not need any battery but harvests energy from the phone WiFi transmissions. The iPoint delivers information to the mobile phone through a low power LCD display that can be captured and processed by a software application. We introduce a mechanism of Packet Width Modulation (PWM) to encode the phone requests in the length of WiFi packets. This allows the use of phones not equipped with RFID readers, and still allows the ultralow power microcontroller to decode the information. In this paper, we describe the architecture of iPoint, discuss the design choices of each component, and report on the experimental evaluation of our prototype. Various RadioFrequency energy harvesters are discussed and a WiFi tailored, modified Greinacher voltage multiplier, a highly efficient parallel full-wave rectifier, is designed, prototyped and fully characterized. It features an energy-harvesting efficiency of up to $72 \%$ and collecting up to $2.5 \mathrm{~mW}$ from a phone WiFi transmission. An ultralow-power micro-controller with LCD capability (TI MSP430F417) is optimized, and customized to interface with our RF Front End (RF-FE). A PWM demodulator is designed, as an integral part of the energy-harvester, and interfaced with the microcontroller. Finally, the mobile phone application for decoding the LCD output is presented. The evaluation is based on actual measurements carried on the third generation of prototypes we built.
\end{abstract}

\section{INTRODUCTION}

Providing information anytime, anywhere, to anybody is a challenging task. Consider an application where we want to deliver information to any person equipped with a standard smartphone in even remote locations where there is no network coverage, and where no source of energy is available. The information should be delivered from a device that can last decades without maintenance. Examples, of applications include information delivery to hikers lost in the woods (e.g., directions, closest points for assistance), caves, and also highdensity and interactive information tags (e.g., tourists information, museums). We explore various technologies, introduce new communication paradigms and build a prototype of such a system that we call iPoint.

In the process of designing the iPoint, we focussed on five key characteristics:

- Universality: the device should be capable of interacting with any smartphone, equipped with the commonly

\author{
Guevara Noubir \\ College of Computer and Information Science \\ Northeastern University \\ Boston, MA 02115 \\ Email: noubir@ccs.neu.edu
}

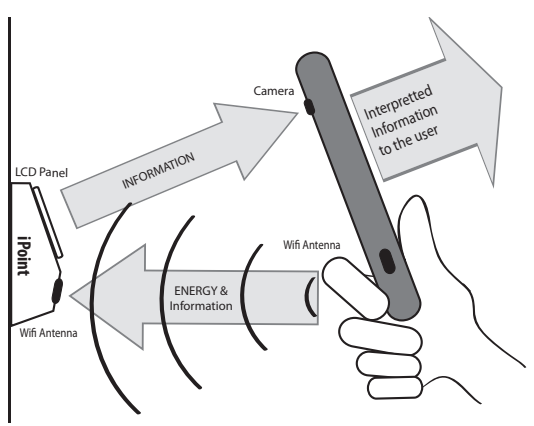

Fig. 1. Outlook of the concept.

available WiFi interface and camera, without hardware modifications. Installing a software application should be sufficient to enable the ad hoc interaction.

- Longevity: the device should be able to last forever without having to rely on a battery, or other depletable or unpredictable sources of energy (e.g., solar). It should draw all its energy from the interacting smartphone.

- Efficiency: the device should consume as little energy as possible for both receiving, computing, and transmitting.

- Interactivity: the device should be able to accept, process, and reply to specific requests from the user.

- Sturdiness: the device should be robust to last forever and therefore should not have moving part or mechanically active components such as interfacing wires or connectors.

These defining features led us to a design that introduced innovative communication paradigms and techniques and the integration of a set of fairly unrelated technologies. In the following, we review the system hardware and software architecture, the components, and the communication paradigms and techniques:

- iPoint components: the iPoint consists of an RF-energy harvester optimized for the $2.4 \mathrm{GHz} \mathrm{WiFi}$ band (collects upto $2.5 \mathrm{~mW}$ power from a WiFi transmission and $300 \mu W$ for a typical use of the iPoint data encoding application), an integrated packet width demodulator, an ultralow-power microcontroller (TI MSP43F417 consuming $40 \mu \mathrm{W}$ when operating at a $8 \mathrm{KHz}$ frequency) capable 
of driving a multi-segment LCD display with extremely low energy cost.

- Smartphone: virtually any smartphone with a WiFi network interface and an integrated camera. The only constraint is that the smartphone should allow the download of applications which is the case of most phones today (e.g., android, iphone, j2me capable phones).

- Energy-provisioning: the smartphone can transfer energy to the iPoint through electro-magnetic radiation using the WiFi interface. This is somewhat similar to the operation of an RFID, without requiring a hardware modification to the phone to integrate an RFID reader. However, this results in severe constraints such as the limited efficiency of energy transfer at higher frequencies, and the limited transmission power of smartphones (typically in the milliwatts using a small antenna instead of several watts of an RFID reader using a relatively larger antenna).

- Multimodal communication: we propose two novel communication mechanisms to circumvent the severe energy asymmetry and constraints. 1) The information from the smartphone to the iPoint is encoded in the WiFi packet width, this drastically reduces the complexity and energy cost of information demodulation at the expense of a significantly lower datarate. 2) The information from the iPoint to the smartphone is encoded on an multi-segment LCD display requiring significantly less energy from the iPoint than a traditional RFID.

In Section III, we present the architecture of iPoint, key components, and communication techniques. In Section IV, we present and discuss the performance of our iPoint prototype. In Section V, we discuss direction for future work.

\section{RELATED WORK}

RFID tags have the potential to deliver information anytime, anywhere [1], [2]. However, RFID tags have significant limitations making them impractical for delivering a substantial amount of information to commodity smartphones. First of all, equipping smartphones with an RFID reader is a significant and challenging modification to the phone hardware. Secondly, among the three types of RFIDs (i.e., passive, active, and semi-active), only the passive ones do not require a battery and therefore satisfy the longevity constraints of the iPoint. However, passive RFIDs require the readers to transmit at high power (in the order of watts), with large antennas. Furthermore, such RFIDs are only capable of storing a very limited amount of information (e.g., 128 bytes) and are not capable of sophisticated interactions.

Recently, a clever alternative solution to RFID tags and traditional barcodes, called bokode, was developed to deliver information from a dot of 3 millimeters diameter encapsulating a high density Data Matrix code [3]. The information is revealed by putting an off-the-shelf camera in an out of focus mode. This solution has the advantage to reduce the size of the tag and increase the information density but still keeping the tag passive. However, bokode still lacks a two-way communication capability and requires sophisticated digital cameras (10Megapixel with a large lens) with in/out focus capability. In the future, if smartphones become equipped with controllable focus cameras, the envisioned iPoint system might benefit from integrating a bokode-based LCD display to deliver information to a smartphone at a lower energy cost.

Several RF-energy harvesting techniques and prototypes were explored over the last few years. The WISP platform and its variants harvest energy from RFID reader [4], TV radio stations [5], and are capable of powering a similar ultralowpower microcontroller as the one used in our iPoint prototype. The WISP was also used as a batteryless sensor node to communicate with a traditional RFID reader [6]. It relies on a high energy sources $(30 \mathrm{dBm})$ operating at a medium RF frequency $915 \mathrm{MHz}$. The constraint of the iPoint to operate on the low RF energy from smartphones (few $\mathrm{dBm}$ ) and higher WiFi frequency (i.e., $2.4 \mathrm{GHz}$ ) requires more advanced RFenergy harvesting mechanisms that we present in the next sections. Other platforms for wireless power transfer exist but require either high transmission power on $915 \mathrm{MHz}$ band [7], or require highly customized transmitters and receivers such as in wireless power transfer via strongly coupled magnetic resonances [8].

\section{SYSTEM ARCHITECTURE}

In this section, we discuss the architecture of the proposed system. We start with detailed description of the system components including the required features, important parameters, and trade-offs. Different design choices along with their advantages and disadvantages are discussed. At the end, we elaborate the interfaces and communication mechanisms between each components.

\section{A. iPoint Components}

We break down the design of the iPoint device into the following components: An RF energy harvester front end responsible for receiving wireless information and providing the required energy to the device, and an ultra low power computing core with a display to process the information and show the outputs.

1) $R F$ energy harvester front end: As its main feature, the RF energy harvester front end efficiently harvests the energy of the received WiFi signals from the Smartphone. In addition, the front end will perform a demodulation on the received signal and sends the information to the computing core.

Rectifier: To collect the energy, the received WiFi signal from the antenna is rectified. The rectification process takes an alternating current/voltage (in this case a $2.4 \mathrm{GHz}$ wave) and converts it into a direct current/voltage that can be accumulated into capacitors. Several types of rectifier circuits have been designed to perform such task, each of which presents its own advantages and disadvantages. In our system, 


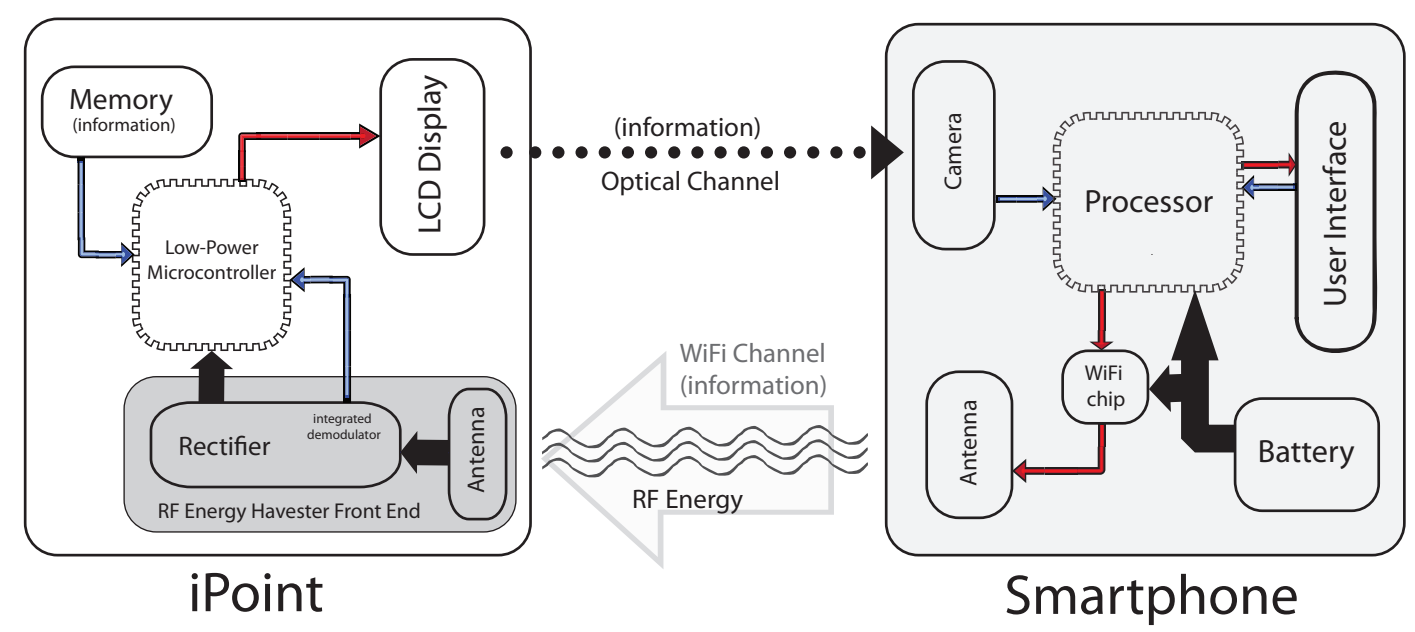

Fig. 2. Detailed diagram of the system.

the input signal of the front end is a very low power WiFi signal with an amplitude much lower than minimum supply voltages for today's active chips (i.e. $1 \mathrm{~V} \sim 5 \mathrm{~V}$ ). Therefore, in order to build a large enough voltage to run the active components, using a multi-stage rectifier (voltage multipliers) is unavoidable. Extremely small input power calls for a high efficiency in the rectification procedure. Using diodes with low forward voltage such as Schottky and low leakage capacitors significantly improves the efficiency of the rectifier. On the other hand, while half-wave rectifiers are simple and compact, they do not show a good efficiency or ripple. Full-Wave rectifiers bring the desirable high efficiency and smaller output ripple by operating on both phases of the input signal. However, a full-wave rectifier requires a differential output design. Considering the characteristics of the system, a multi-stage full-wave rectifier such as Greinacher multiplier is a reasonable choice [9].

Integrated Demodulator: Having investigated different mechanisms for demodulating the information in the received signal, we found out that the output of the early stages in the multi-stage rectifiers represents the envelope of the input signal provided the intermediate capacitance of the rectifier is small. Assume the input signal is a train of WiFi signal bursts with information encoded in length of the bursts (See Figure 3). Therefore, the envelope signal can be used as a partially demodulated information fed to the computing core of the system. A passive external circuit will adjust the amplitude level of the signal to make it readable by chips powered by the rectifier. This is discussed in detail in Section IV.

Matching: The antenna and the rectifier should be carefully matched in the frequency of the operation which in the case of iPoint is one of the standard WiFi channels. This is done by using a matching network between the antenna and the rectifier. Normally, the values for the matching network are calculated experimentally. Note that there is always a trade-off between having a better match and the bandwidth of the system. Hence, the matching network should be optimized to get the maximum power from the WiFi signal with the bandwidth of approximately $22 \mathrm{MHz}$.

Regulatory Circuit: In some cases, a rectifier can build an excessively large output voltage. This may be destructive to other iPoint components. In order to avoid any possible harm, the output voltage of the rectifier is connected to a voltage regulator.

In our prototyped device, we used a modified 10-stage Greinacher multiplier tuned on WiFi channel $1(2.412 \mathrm{GHz})$. The front end shows an efficiency up to $82 \%$. We provide more details on the design and its performance in Section IV.

2) Computing Core: The Computing Core of the iPoint receives the demodulated request from the integrated demodulator of the front end, processes it, and sends the correspondent output to the display. This component is the active part of the device. The required power for the operations is obtained from RF Energy harvester front end. Due to the very restricted budget of the energy, the core must function in ultralow power fashion while providing moderate computing capabilities. This makes the use of microcontrollers (MCUs) a preferred design choice in this case. Ultralow power MCUs such as TI MSP430 series are designed and optimized for low power embedded devices and have the desired computing power including LCD driver. We lowered the clock frequency of the MCU in order to reduce the power consumption even further.

The display power consumption should be minimized at the same time. LCD displays require very little energy consumption compared to other display panels like LED thus would be a very good candidate for the design. The main factor in LCD energy consumption is the size of the LCD. Larger LCD panels need higher voltages for providing the 
same contrast on the panel. This translates into a higher voltage requirement for the MCU LCD driver, and a higher supply voltage, which results in higher power consumption of the core.

In the prototyped system, we used a TI MSP430F417 MCU with the capability of driving 96 segments of LCD. We underclocked the MCU to $8 \mathrm{KHz}$ to minimize the power consumption of the core $(<50 \mu W$ while driving the LCD display). This is well below the capabilities of the RF energy harvester. More details about the performance of the design are provided in section IV.

\section{B. Smartphone component}

In order to achieve the desired universality for the system, we kept this part as simple as possible. The iPoint system works with any smartphone equipped with a camera and a WiFi Interface. The only component of the system added to the smartphone is the application installed on the phone. Obviousely the phone should allow downloading applications. A suitable API is necessary to give the application a moderate control over the WiFi interface if needed. There will be no change to the hardware of the phone. Installed application allows the user to send different requests to the iPoint, processes and interprets the information received from the device, and interacts with the user by phone's UI (Keyboard, touch screen, etc.).

\section{Communication between components}

Due to severe energy constraints, the components of the system should perform their task and also communicate with one another consuming minimum amount of energy. One possibility to reduce the energy consumption is to keep each module as simple as possible. This demands novel communication mechanisms between different components. In our system, we introduce two different communication channels: Smartphone to iPoint (WiFi), and iPoint to smartphone (Optical).

1) Smartphone to iPoint (WiFi) channel: In the $\mathrm{WiFi}$ channel, information is encoded in and transferred by WiFi packets. Because of the limited energy budget of the iPoint device, implementing an active WiFi demodulator is not feasible. We propose Packet Width Modulation (PWM), a novel modulation scheme that allows us to demodulate the information with the integrated demodulator in the front end. This significantly reduces the complexity of the demodulator and energy consumption of the decoding processing at the price of lower rate of communication. PWM also makes the encoding feasible from any smartphone with a socket network API.

PWM Encoding: In PWM, information is encoded in the length of the transmitted $\mathrm{WiFi}$ packets by the smartphone. Each length represents a specific symbol in the code. A message is defined as a sequence of $\mathrm{WiFi}$ packets. In $\mathrm{WiFi}$ communication, packet width is determined by the size of the packet, rate of communication, and fragmentation.

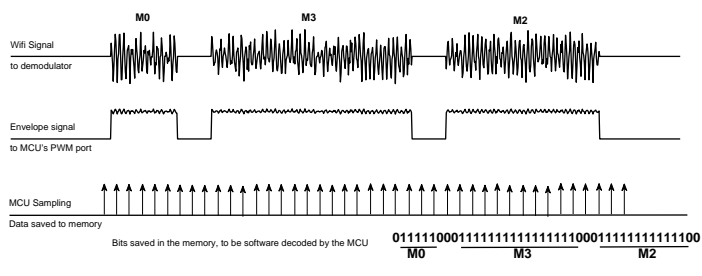

Fig. 3. PWM decoding.

To simplify the encoding process, we configure the WiFi interface of the smartphone to send out packets at a fixed rate. Furthermore, the $\mathrm{WiFi}$ fragmentation threshold is set to the maximum possible to prevent any interference with the PWM encoding process. Since the receiver side (i.e., iPoint) does not have the transmission capability, it sends no acknowledgement packets back. This applies both for the link layer (WiFi) and transport layer. We therefore use broadcast packet to prevent WiFi retransmissions. We also limit ourself to UDP packets since the one-way nature of this channel rules out using TCP in the transport layer but also to be able to control the timing for packets transmissions. Assuming the PWM mechanism uses $M$ different packet lengths, each packet encodes $\log _{2} M$ bits of information.

PWM Decoding: It is possible to retrieve the length of the received packets simply by detecting the envelope of the signal. Note that a single stage of the RF front end's rectifier is essentially an envelope detector circuit. Because of the small capacitance of the intermediate capacitors, we can obtain a quite accurate envelope signal. The first stage presents the best ripple characteristics as it sees the largest amount of the load. Therefore, we use the output of the first stage of the rectifier as the partially demodulated input signal. The partially demodulated signal is connected to the MCU after passing a level-adjustment external circuit. The level-adjustment circuit is a passive voltage shifter that sets the 0 and 1 level of the signal in the readable range for the MCU. MCU samples the PWM signal with an appropriate frequency and processes them after the message is finished. For the MCU to be able to recognize the beginning and the end of the message, we use predefined start and stop Flags.

Obviously, the smartphone needs to send WiFi packets after the end of the message in order to provide the energy required for the iPoint to process the information and send the reply message back through the optical channel.

PWM transmission rate analysis: Let $M$ denote the number of the packet lengths in the PWM. Assume the transmitter sends the packets at the rate, $R$. Let $S_{\min }$ be the smallest packet size. $S_{\text {min }}$ is determined by the MCU clock to guarantee packet detection and length estimation, and by the energy harvester efficiency and MCU energy 
requirements. We consider packets of size multiples of $S_{\text {min }}$, $S_{i}=i \times S_{\min }$. Hence, the average size of the packet would be $S_{a v g}=\frac{S_{\min }+S_{\max }}{2}=\frac{(M+1)}{2} S_{\min }$. Further assume a message is a sequence of packets separated by idle periods of the length equal to $S_{i d l e}$. We can calculate the time needed to send a packet, $T_{p}$ as following:

$$
T_{p}=\frac{S_{\text {avg }}+S_{\text {idle }}}{R}=\frac{(M+1) S_{\text {min }}+2 S_{\text {idle }}}{2 R}
$$

Each packet encodes $\log _{2}^{M}$ bits of information. Therefore,

$$
R_{P W M}=\frac{\log _{2}^{M}}{T_{p}}=\frac{2 \log _{2}^{M} \cdot R}{(M+1) S_{\text {min }}+2 S_{\text {idle }}}
$$

The values of $S_{\min }$ and $S_{\text {idle }}$ are determined by the maximum sampling rate of the MCU at iPoint side. Assuming $F_{M C U}$ is the sampling frequency of the MCU, we have,

$$
S_{\text {min }}, S_{i d l e}>\frac{2}{f_{M C U}} \text { (Nyquist theorem) }
$$

Hence,

$$
\begin{aligned}
R_{P W M}(\max ) & <\frac{2 R \log _{2}^{M}}{(M+1)\left(\frac{2}{f_{M C U}}\right)+2\left(\frac{2}{f_{M C U}}\right)} \\
& =\frac{\log _{2}^{M} \cdot R \cdot f_{M C U}}{M+3}
\end{aligned}
$$

There are a few more constraints in the design of the system. One might have noticed that during idle periods, iPoint is not receiving power from the smartphone. This lowers the output voltage of the rectifier which may result in unwanted shut-down of the system. To maintain the harvested level above the desired threshold, $S_{\min }$ needs to be larger than $S_{\text {idle. }}$. We define the duty cycle of the system as the $\frac{S_{\min }}{S_{\text {idle }}}$ ratio. The minimum duty cycle that allows the system to operate continuously, depends on the implementation and may be evaluated experimentally. Moreover, $S_{\max }=M \times S_{\min }$ should be smaller than the fragmentation threshold in the smartphone's WiFi interface.

2) iPoint to smartphone (Optical) channel: The smartphone's transmitting power is much lower than a conventional RFID reader (few milliwatts for the smartphone versus few watts for the RFID reader). Therefore, using a similar scheme as passive RFID tags (i.e. back scattering) is not practical. Instead, iPoint uses a low cost way of sending information to the smartphone taking advantage of the imaging and computing capability of the phone. After processing the request sent from the user, iPoint sends the corresponding information via the optical channel. The information is encoded in series of LCD segment patterns. The smartphone captures the sequence of the patterns with the camera, recognizes the patterns, interprets the information, and finally sends the interpreted data to the user through its own UI. It is worth noting that all the expensive operations are done on the smartphone side. This leads to a significant drop in the encoder/transmitter complexity of the iPoint. This also proves to be a very energy efficient method as displaying information on the LCD panel requires far less

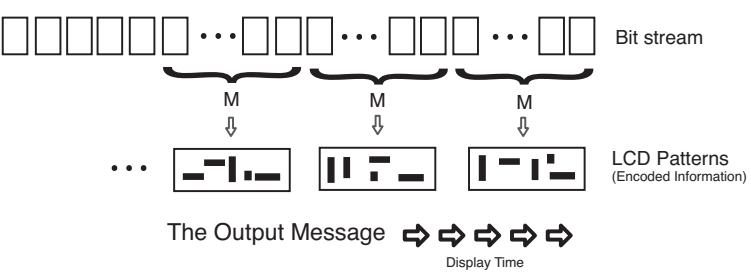

Fig. 4. LCD Pixel Encoding for a $M$-segment LCD panel.

energy compared to conventional back scattering scheme used in passive RFID tags. To get an intuition about the energy efficiency of LCD displays, one can think of the lifespan of wrist watches with LCD display; they run for years on a tiny button cell holding a small amount of charge.

LCD Pattern Encoding (LPE): Let a LCD pattern denote a combination of the LCD display's segments where a segment can be ON or OFF. Upon receiving a request, the MCU computes the output message for the LPE, which is defined as a sequence of distinct LCD patterns. The MCU is programmed in advance to run the computation instructions. Finally, the MCU displays the output message pattern by pattern on the LCD panel. An output message which consists of $n$ patterns on a $M$-segment LCD panel, encodes $n \times M$ bits of information.

LCD Pattern Decoding (LPD): At the other end of the channel, the LPE message is captured by the smartphone's camera. This can be done by recording a video or taking a series of pictures at a specific rate. The phone decodes the captured message by running a simple pattern recognition algorithm on each frame. Lastly, the interpreted data is sent to the user via UI or used in the next sessions of communication.

LPD transmission rate analysis: The LCD pattern update rate can go up to $200 \mathrm{~Hz}$. However, our experiments indicate that the deciding factor on an error free decoding is the sampling rate of the camera. Let $R_{c}$ denote the maximum sampling rate of the smartphone's camera. Applying Nyquist theorem we have,

$$
R_{\max }<\frac{R_{c}}{2} \text { fps. }
$$

Therefore, for a $M$-segment LCD display, we have the upper bound of $\frac{R_{c} M}{2}$ bps. For most of today's commercial smartphone cameras, $R_{c}=25 \mathrm{fps}$.

In the prototype, we utilize a simple pattern recognition algorithm based on applying a detection mask on each captured frame. To simplify and speed-up the detection process, the mask-frame alignment is done by the user. Section IV discusses the design in more detail.

\section{PRototype AND}




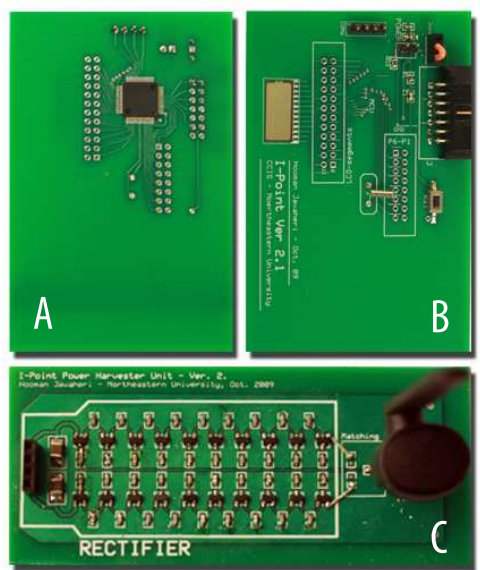

Fig. 5. The iPoint prototype boards: A) Computing core upper layer (LCD display is shown) B) Computing core lower layer (MSP430 is shown) C) RF energy-harvester front end, 10-stage Greinacher voltage multiplier.

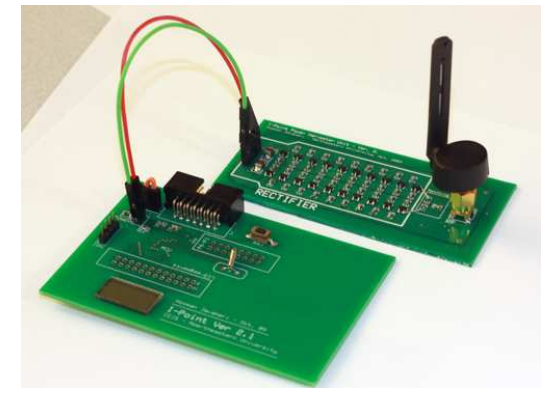

Fig. 6. The realization of the iPoint.

\section{PERFORMANCE EVALUATION}

We prototyped a version of the iPoint based on the design described in Section III. The device is shown in figures 5 and 6. In this section, we explain the implementation of the components in detail. Several experiments are carried out in order to accurately characterize the prototyped device and prove the functionality of the design components. This section presents the detailed description of the testbed and experimental results.

\section{A. Smartphone}

The smartphone used in the experiments is the HTC Dream also known as T-Mobile G1 with Android mobile device platform Ver. 1.6. This $3 \mathrm{G}$ phone is equipped with a $528 \mathrm{MHz}$ Qualcomm ARM11 Processor, 192 MB of DDR SDRAM, $320 \times 480$ pixel LCD Display with 180 ppi, 3.2 megapixel camera with auto-focus capability, and a WiFi $(802.11 \mathrm{~b} / \mathrm{g})$ wireless interface [10].

\section{B. Software application}

A user application was developed for the prototype. This program sends the different requests modulated with the proposed PWM scheme. The WiFi interface of the smartphone was configured to send the packets at a fixed

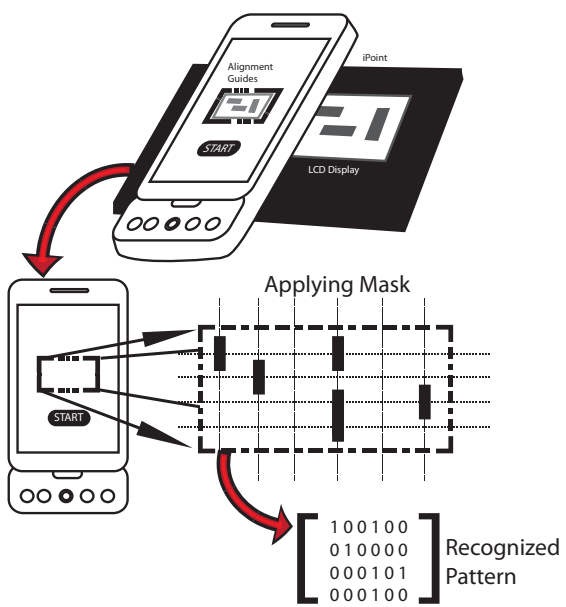

Fig. 7. The pattern recognition, Sampling of the frame is performed only on the intersections of mask grid-lines (Narrow dashed lines).

rate of $1 \mathrm{Mbps}$, the lowest rate for WiFi communication. This has the advantage of preventing the $\mathrm{WiFi}$ auto-rate functionality from interfering with the PWM mechanism. It also minimizes the idle time, therefore delivering more energy to the energy-harvester. Ideally, the application should create an ad hoc network and send the broadcast UDP packets. However, the Android platform support for the ad hoc mode is currently limited. As a temporary alternate solution, a WiFi network created by an external Access Point was used by the smartphone to send the information. Note, that is not a roadblock to the iPoint concept, since other mobile operating systems provide adequate support for the ad hoc mode (e.g., Windows Mobile) and Android will most probably do the same in the near future.

A session of the program can be described as following: For the LPD, the program applies a very simple pattern recognition scheme. The application shows the live capture of the camera on the screen. As illustrated in figure 7, there is a virtual guide on the screen that helps user to align the camera and LCD display of the phone. Having aligned the LCD panel capture with the virtual guide, the user starts the application and chooses to send a request. This triggers the PWM encoder that encodes the request with PWM mechanism and sends it over the WiFi interface. At the same time, the camera starts recording the capture and saves the frames. Since the user already aligned the LCD panel, the LPD decoder samples a predefined points on each capture and decodes the information based on ON segments on each frame. Finally, the interpreted results are shown on the screen.

\section{C. iPoint Hardware}

1) RF energy harvester Front end performance evaluation: As depicted in Figure 8, the implemented rectifier is a 10-stage modified Greinacher voltage multiplier. This, basically is a full-wave rectifier built from two mirrored half-wave rectifiers connected to the same RF input. This 


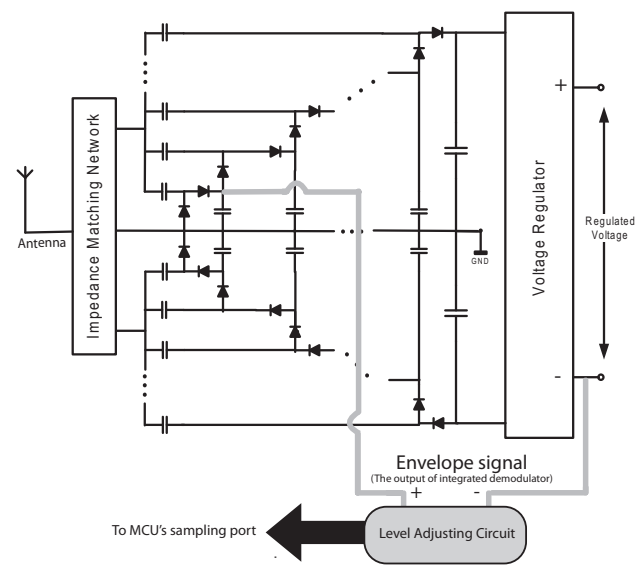

Fig. 8. RF Energy Harvester Front end including integrated demodulator.

design gives us a double output voltage and improves the ripple characteristics of the device. Since the rectifier and the computing core do not need to share a common ground, there is no need for a differential design for the interface. We simply ground the negative output of the rectifier. To maximize the performance of the rectifier, we use high quality low-leakage RF capacitors (Cap names here) and low-threshold Schottky diodes (HSMS-282 series from Avago Technologies). These Schottky diodes have the lowest forward voltage $(150 \sim 200 \mathrm{mV})$ among all bulk diodes available in the market and are optimized to work in the frequencies above $1.5 G H z$ [11]. The intermediate capacitors are chosen small (few picofarads) to maximize the output voltage level. in contrast, a very large load capacitance in the order of $10 \mu \mathrm{F}$ is installed to reduce the ripple and big voltage drop on the load.

The rectifier was matched on WiFi channel $1(2.412 G H z)$ experimentally (using a network analyzer) with an LC matching network. In our approach, the rectifier is tuned until the output voltage is maximized in the frequency of operation. Note that the parallel RF input will reduce the input impedance of the circuit and makes the matching procedure easier to deal with. The frequency response of the circuit obtained from the network analyzer before and after matching is illustrated in Figure 9.

In order to characterize the efficiency of the energyharvester, a MXG Vector Signal Generator was used to feed the rectifier and the output voltage level of the energyharvester was measured. The signal generator was connected to the energy harvester via a 0.5 feet coaxial cable. The energy harvester was fed with a WiFi signal in a wide range of input power, from $-20 \mathrm{dBm}$ to $15 \mathrm{dBm}$. The output voltage and efficiency were measured without a load and with a load of $140 \mathrm{~K} \Omega$. This $150 \mathrm{~K} \Omega$ load was chosen because it corresponds to the MCU impedance in active mode. The output voltage is presented in Figures 10 and 11.

Integrated PWM demodulator: In order to test the

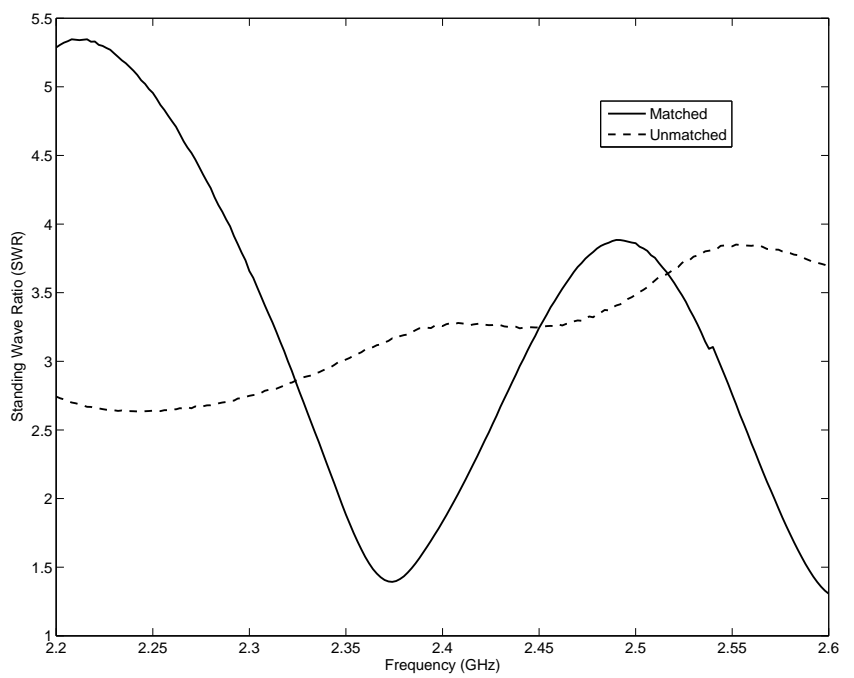

Fig. 9. SWR values for the rectifier circuit before and after matching. The data obtained from a E5062A ENA Series network analyzer from Agilent Technologies.

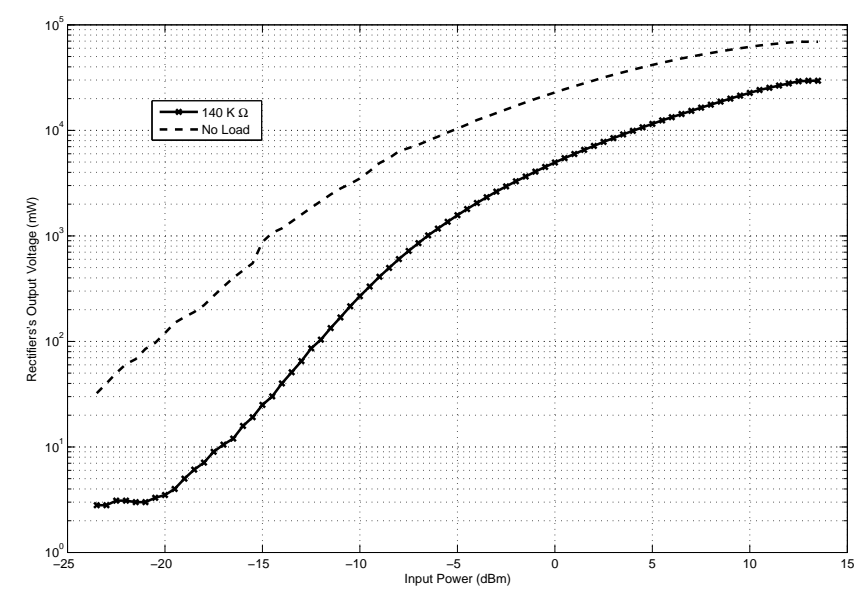

Fig. 10. Performance of the energy harvester unit.

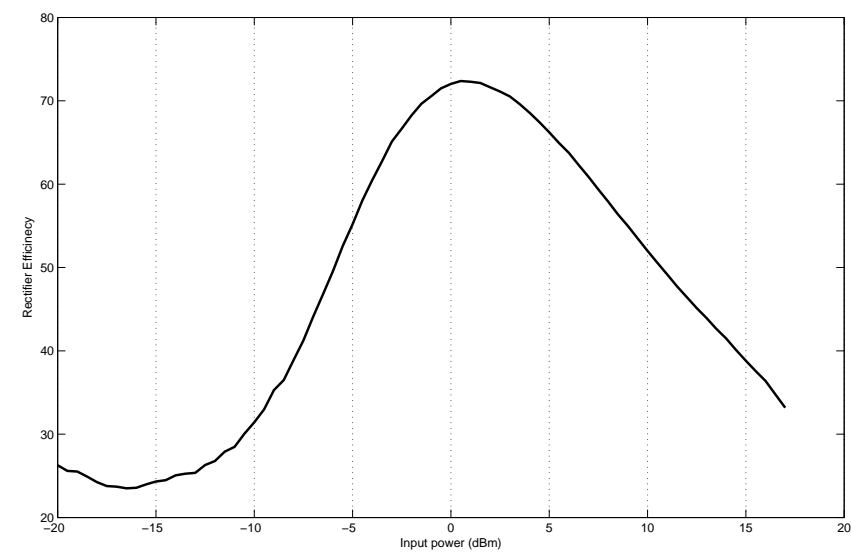

Fig. 11. Energy-harvester efficiency as a function of input power. 


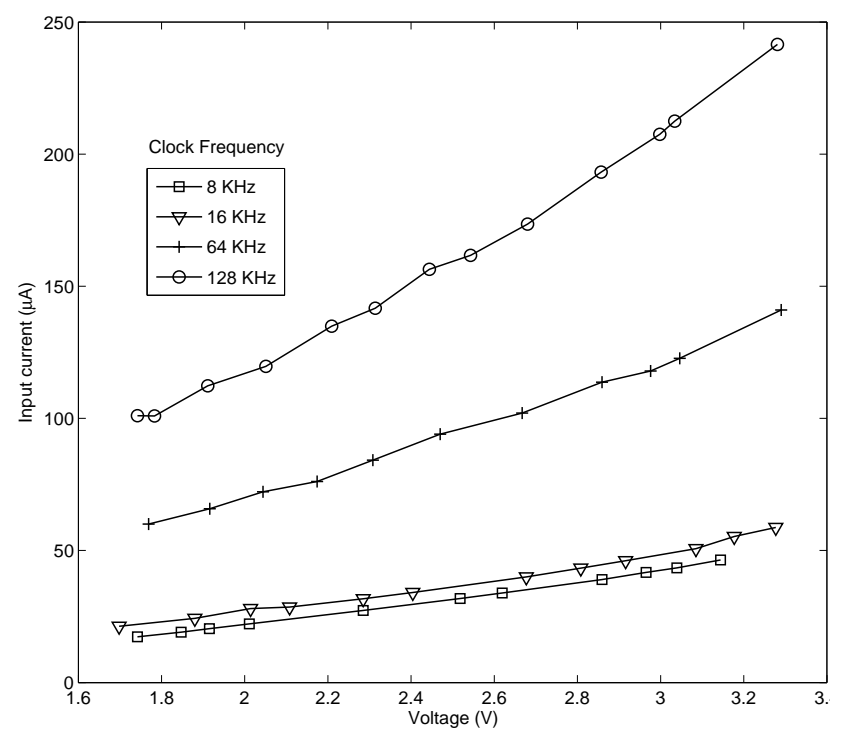

Fig. 13. Power consumption of MCU for different clock frequencies.

functionality of the integrated demodulator of the front end, packets with different sizes were sent over the WiFi channel by the smartphone while the output of the energy harvester and integrated demodulator being measured. The rate of communication was fixed to $1 \mathrm{Mbps}$. The result is shown in Figure 12.

2) Computation core performance evaluation: For the communication core, we embed a TI MSP430F417, an ultralow power microcontroller from Texas Instruments. This 16-bit flash MCU provides our desired computing capabilities at low power consumption. It features $32 \mathrm{~KB}+256 \mathrm{~B}$ of flash memory, $1 \mathrm{~KB}$ of RAM, Low supply voltage of $1.8 \mathrm{~V}$, integrated LCD driver for 96 segments, on-chip comparator that can be used for finalizing the PWM signal demodulation, and very low active power consumption of $200 \mu \mathrm{A}$ at $1 \mathrm{MHz}$, which makes it a preferred choice for the iPoint prototype.

The power budget of the energy harvester is extremely limited due to the small transmission power of the smartphone. Meeting the requirements of this very low power scenario requires the MCU to reach its minimum power consumption. One possibility to reduce the power consumption of the MCU is to lower the clock frequency. Since the operations of the iPoint's computing core do not demand a very fast clock, it is possible to reduce the clock frequency to a few kilohertz. This is an area that differentiates the iPoint design from other approaches in low-power embedded computing which require that the MCU operates at much higher frequencies (i.e., few megahertz) [4]. Figure IV-C2 illustrates the results of our measurements of the power consumption of the MCU running the same instructions at different clock frequencies.

Minimum duty cycle as one of the important characteristics of the iPoint system was discussed in section III. An

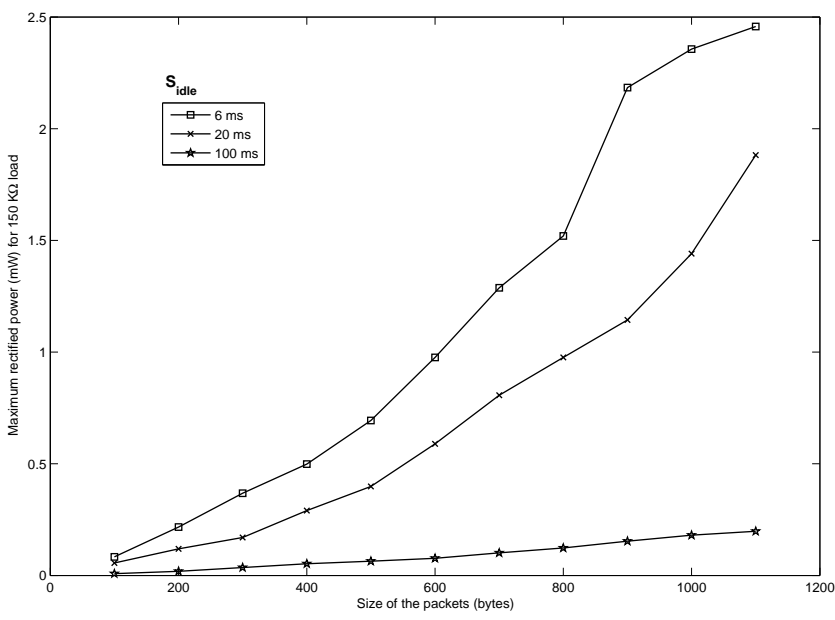

Fig. 14. The rectified output voltage as a function of the packet length for different idle times $S_{\text {idle }}$ (therefore duty cycles).

experiment was performed to evaluate the minimum duty cycle of the prototyped iPoint device. The results of the experiment are summarized in figure 14 .

LCD Display: The LCD panel selected for this generation of prototyped device is a 26 segment watch LCD display. For this selection, the power consumption of two LCD panels with different sizes were measured. Panel $1(1.5 \mathrm{~cm} \times 3 \mathrm{~cm}, 24$ segment), and Panel $2(1 \mathrm{~cm} \times 1.8 \mathrm{~cm}, 26$ Segments). A test image was taken from the LCD panels at the same distance and under the same light environment while the same pattern were displayed on both panels. The contrast of the panels were compared digitally in Photoshop. It was shown that the larger LCD needs $2.9 \mathrm{~V}$ to create the same contrast as LCD panel 2 does being driven by $1.9 \mathrm{~V}$. The input current of the boards were measured $49 \mu \mathrm{A}$ and $21 \mu \mathrm{A}$ respectively.

\section{IMPROVEMENTS AND FUTURE WORK}

In the short term, we will investigate the channel capacity, and BER for both the Packet Width Modulation and Optical feedback. We will propose error correction codes specially designed for these two channels. We also plan to investigate the adaptation of the bokode concept to utilise an ultralow power LCD display with high information density.

\section{CONCLUSION}

We introduced iPoint, a device that can interact with commodity smartphones, equipped with a WiFi network interface and camera, therefore enabling ad hoc and universal communication. At the core of iPoint lies an ultralow power microcontroller. iPoint draws the integrality of its energy from the smartphone transmissions, through an RF energy-harverster, making the use of batteries unnecessary and guaranteeing its longevity. Two new communication paradigms are introduced: 1) Packet Width Modulation allows the smartphone to encode information in the width of the WiFi packet and making 


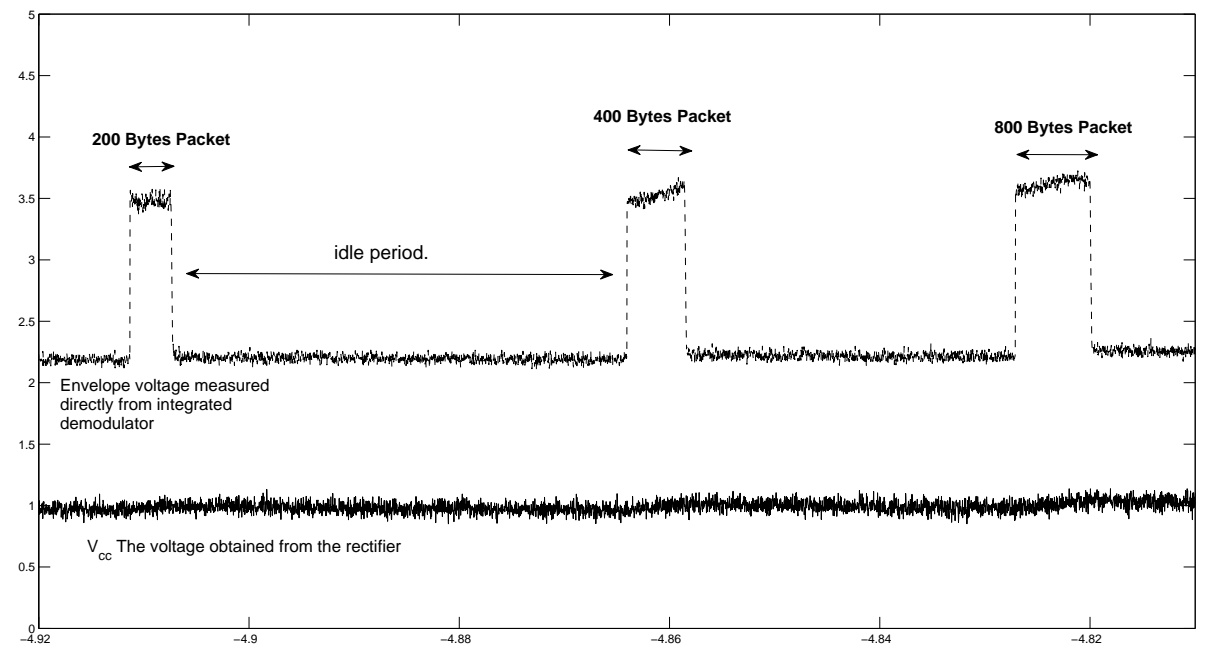

Fig. 12. The outputs of Energy harvester ( $V_{c c}$ and integrated demodulator (envelope signal). Note that the size of the packet is easily detectable. The WiFi communication rate was fixed to $1 \mathrm{Mbps}$. harvester's output level is fairly smaller than the peaks of envelope signal. That reason is the long idle times between packet transmissions. The data is captured by a Infinium MSO8104 oscilloscope from Agilent Technologies. The plot is regenerated in MATLAB.

demodulation extremely energy efficient, 2) LCD information encoding and camera decoding. We discussed several design possibilities and built a prototype of the iPoint. We reported on the performance of our system for various transmission powers, operation frequencies of the microcontroller, packet sizes and duty cycles.

\section{REFERENCES}

[1] R. Weinstein, "Rfid: A technical overview and its application to the enterprise," IT Professional, vol. 7, no. 3, pp. 27-33, May 2005.

[2] EPCglobal, "Epcglobal standards and technology," 2008, http://www.epcglobalinc.org/standards/.

[3] A. Mohan, G. Woo, S. Hiura, Q. Smithwick, and R. Raskar, "Bokode: imperceptible visual tags for camera based interaction from a distance," in SIGGRAPH '09: ACM SIGGRAPH 2009 papers. New York, NY, USA: ACM, 2009, pp. 1-8.

[4] A. Sample, D. Yeager, P. Powledge, and J. Smith., "Design of an rfidbased battery-free programmable sensing platform," IEEE Transactions on Instrumentation and Measurement, 2008.

[5] A. Sample and J. R. Smith, "Experimental results with two wireless power transfer systems," in IEEE Radio and Wireless Symposium (RawCon 2009), 2009.

[6] M. Buettner, B. Greenstein, R. Prasad, A. Sample, J. R. Smith, D. Yeager, and D. Wetherall, "Demonstration: Rfid sensor networks with the intel wisp," in 6th ACM Conference on Embedded Networked Sensor Systems (Sensys'08).

[7] "Powercast corporation," http://www.powercastco.com/technology/powerharvesterreceivers.

[8] A. Kurs, A. Karalis, R. Moffatt, J. D. Joannopoulos, P. Fisher, and M. Soljacic, "Wireless power transfer via strongly coupled magnetic resonances," Science, 2007.

[9] J.-P. Curty, M. Declercq, and C. Dehollain, Design And Optimization Of Passive UHF RFID Systems. Springer, 2007.

[10] "Htc dream," http://www.htc.com/www/product/dream/overview.html.

[11] "Avago technologies, surface mount rf schottky barrier diodes," http://avagotech.com/pages/en/rf_ics_discretes/schottky_diodes/surface_mount/. 